\title{
The effects of stochastic market clearing on the cost of wind integration: a case of New Zealand electricity market*
}

\author{
Javad Khazaei and Golbon Zakeri and Geoffrey Pritchard
}

April 12, 2013

\begin{abstract}
Introducing wind generation into an electricity market can incur an extra cost resulting from the volatile nature of wind. To reduce this cost, an alternative stochastic market clearing mechanism is proposed in the literature $[1,2,3]$. However, implementing a stochastic market clearing can also impose some extra cost on the market. Therefore, it is essential to estimate the efficiency gain resulting from implementing a stochastic market clearing mechanism. We describe the result of an empirical study to quantify value of a stochastic clearing mechanism for the New Zealand electricity market. We extend our analysis for possible larger wind integration in the future.
\end{abstract}

\section{Introduction}

The wholesale New Zealand electricity market started operating in October 1996. The current market clearing mechanism has a pre-dispatch stage two hours prior to real time realization of demand and hence generation. This is designed to give the generators an estimation of what they will be asked to produce in the spot market. At the time of the pre-dispatch, real demand and generation from intermittent sources such as wind are unknown. Thus, the real generation quantities can be different from pre-dispatch values. As uncertainty is revealed only in real-time, the spot deviations are announced within only a few minutes of generation. However, some generators are unable to change output rapidly. Thus, the ISO cannot use these generators for the spot deviations. The other more flexible generators are possibly less efficient (e.g. more costly). Therefore, this approach to deal with uncertainty can cause inefficiency in the electricity market.

On the other hand, by the increase of production from intermittent sources such as wind energy, market volatilities increases. Currently, wind generators produce about $4 \%$ of electricity generation in New Zealand. However, it is expected that wind penetration may increase to $20 \%$ by $2030[4,5]$.

Stochastic Programming market clearing mechanism is one suggested method to clear the market more efficiently in the presence of uncertainty [3, 2]. The Stochastic Programming Mechanism (SPM) is different from the Conventional Mechanism (CM) in the sense that it takes into account the future possibilities in the current decision. Thus, it always improves the expected value of the solution. However, there is also a cost involved in implementing the SPM. Efforts need to be made to generate a distribution function for the uncertain parameters and and we need to solve larger problems to determine dispatch. Thus, if the benefits of implementing the SPM are not large enough, the conventional mechanisms $(\mathrm{CM})$ may be preferred.

\footnotetext{
${ }^{*}$ The authors would like to thank the reviewers for their careful evaluation of the paper and for their helpful comments.
} 
In this paper, we present a model that can be used to approximate the value of the SPM. We implement this model for New Zealand Electricity Market (NZEM). In our experiments, we first produce a pre-dispatch "plan" from each of the conventional and SPM market clearing mechanisms. Then, we proceed to real time and deviate from the "plan" based on minimizing cost of generation as currently happens in New Zealand. Once the deviation has been determined based on order merit (determined through offered cost of generation), we measure the true cost of generation.

In addition to estimating the value of SPM for the current NZEM, we design experiments for the NZEM with future developments, with higher wind integration. This provides an approximation of the value of the SPM for different percentage of wind integration in New Zealand Electricity Market.

\section{The wholesale electricity market}

The New Zealand wholesale electricity market is an energy only exchange. The pricing scheme is a nodal uniform price auction. Since 2004, NZEM has been a compulsory market. All generators larger than $10 \mathrm{MW}$ are required to offer to the wholesale market. Demand entities can also bid into the market. Each day consists of 48 trading periods, and separate bids and offers are made for each trading period. Generators can start offering to the market from 36 hours before dispatch. From 4 hours before dispatch, the ISO calculates and announces a forecast price every half hour, as a guide to the market participants. Offers are locked in two hours in advance of the time period. Market prices are calculated ex-post (i.e. after dispatch) according to the locked-in offers and actual dispatch in each node. The offers are publicly available two weeks after dispatch. The New Zealand Electricity Authority is responsible for publishing these data as a part of a Centralized Data Set (CDS) [6, 7].

Each generator can offer up to five tranches. Each tranche consists of a quantity and a price. This indicates the willingness of the offering generator to produce up to the offered quantity for a price of at least the offered price. All together, these tranches make a piecewise constant function which is called an "offer stack". We represent the offer stack for generator $m$ by $C_{m}(x)[6,7]$.

As currently there is very little demand-side bidding in NZEM, we ignore demand-side bids in our analysis ${ }^{1}$. Thus, we assume demand is inelastic.

The nodal electricity prices are determined by an optimization problem called SPD ${ }^{2}$. An Independent System Operator (ISO) solves this problem for each of the time periods. SPD is a DC-load flow model. Readers can refer to [8] for more information about SPD. The simplified electricity network can be represented as a simple flow model (see fig. 1).

In fig. $1, i, j$, and $k$ represent network nodes. $l_{j, k}$ denotes the flow in the line between $j$ and $k$, from $j$ to $k$. By convention, we assume $j<k$. A negative value for $l_{j, k}$ means electricity flows from $k$ to $j$. We represent the number of nodes by $n$, and $D_{i}$ is the demand at node $i$. For a node $j$, the set $O(j)$ defines all the generating units located at node $j$. Generator $m$ produces $q_{m} \in Q_{m}$. The

\footnotetext{
${ }^{1}$ Wind generation can be thought of as decreasing demand. Hence, volatility in wind generation is equivalent to volatility in demand. Therefore our models are effectively general enough to cope with demand volatility as well, although we have not implemented this in our experiments. Our experiments are based on historical periods. During these periods demand was realized and the CDS has measurements of all nodal demands. Through aggregating these we arrive at our demand figures for the simplified NZ model.

${ }^{2}$ Schedule, Price and Dispatch
} 


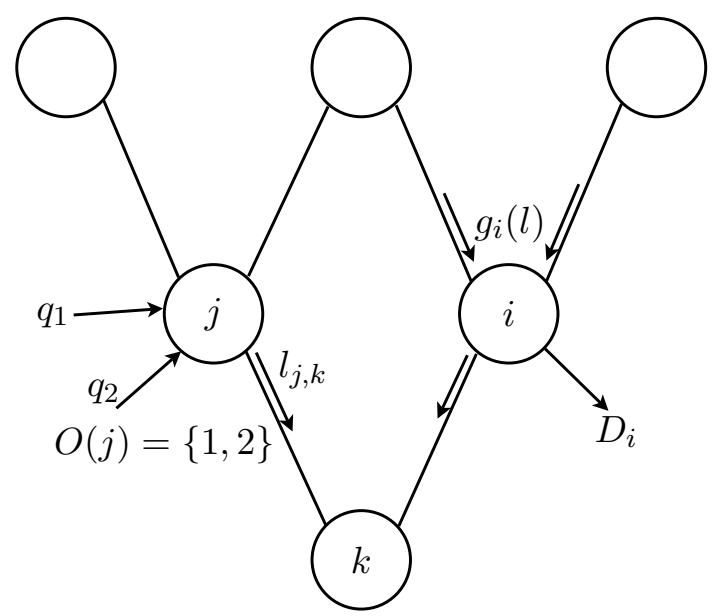

Figure 1: network flow model [6]

simplified SPD model, is then

$$
\begin{array}{cc}
\text { SPD: } \min _{q, l} & \sum_{i} \sum_{m \in O(i)} \int_{0}^{q_{m}} C_{m}(x) d x \\
\text { s.t. } & g_{i}(l)+\sum_{m \in O(i)} q_{m}=D_{i} \\
& , i \in\{1, \ldots, n\}, \\
q_{m} \in Q_{m} \\
l \in L .
\end{array} \quad, m \in O(i), i \in\{1, \ldots, n\},
$$

The constraint $l \in L$ enforces both the thermal limits of the lines and Kirchhoff's Law for loop flow constraints. Accordingly, $L$ is a convex set. The net flow to node $i$ is shown with $g_{i}(l)$. To calculate the net flow, we must also take the line losses into account. For example, with quadratic losses it is

$$
g_{i}(l)=\sum_{k<i} l_{k, i}-\sum_{k>i} l_{i, k}-\frac{1}{2}\left(\sum_{k<i} r_{k, i} l_{k, i}^{2}+\sum_{k>i} r_{i, k} l_{i, k}^{2}\right) .
$$

The dispatch model with this loss function is a non-linear and non-convex optimization problem. To avoid the non-linearity, we have used a piecewise linear approximation of this function in our model similar to the piecewise linear loss models used in the original SPD. This enables us to solve our optimization problems as $\mathrm{LPs}^{3}$.

\section{The models}

For each trading period we solve four models. The first model is the conventional model. It is the simplified version of SPD as described in the previous section. We also use a spot conventional model. This is the optimization problem that is solved after wind realization. It is aimed to seek an optimal

\footnotetext{
${ }^{3}$ This approximation also ensures that our model is compatible with the original SPD formulation.
} 
corrective action. In the spot market, generators are asked to deviate from the already declared predispatch quantity. All generators cannot ramp up or down immediately. Thus, one main new set of constraints is the ramping constraints of generators.

Another model is a stochastic programming model (SP). The stochastic programming model, is an alternative to the pre-dispatch conventional model. The SP model is different from the conventional model in the sense that it foresees a distribution of future possibilities. The SP model minimizes the expected cost of the spot market rather than the pre-dispatch cost based on the realization of a single scenario. Therefore, it mitigates pre-dispatch decisions that lead to expensive corrective actions in the spot market. However, these inefficient pre-dispatch decisions are possible in the conventional mechanism (CM). The fourth model is the spot market for the SPM, which is similar to the spot market of the CM.

\subsection{The conventional model}

The real SPD is a large optimization problem with reserve and security constraints. Over the NZEM, this side constrained network flow problem contains about 250 nodes and approximately 500 arcs. We have chosen a simplified version of SPD which enables us to run our simulation many times. Our conventional model is similar to the model that Philpott et al. [6] have used for their market model over a single period.

The simplified model consists of 18 nodes as represented in fig. 2. This model is small and is confined to the major New Zealand nodes. As a result, the network is a simplified aggregate of the the true New Zealand network which means a number of lines are omitted from the from the model and only appear in aggregate. Therefore the simplified model incurs less loss in it than there would be in reality. To compensate for this, we scaled the demand. Based on a number of experiments, a figure of $5 \%$ increase in the demand yields a good match between the simplified model and SPD. Furthermore we note here that we use the same simplified model as the basis of our conventional model and that used for the stochastic programming market clearing. Therefore much like "common random numbers" technique in variance reduction, we base our simulation experiments on the same models and this would yield a bona fide comparison for the SPM and CM. We assume a large Value of Lost Load (VOLL) which reflects our assumption of inelasticity of demand ${ }^{4}$ in our conventional pre-dispatch model:

$$
\begin{array}{rlr}
\mathrm{CP}: \min _{q, l} & \sum_{i} \sum_{m \in O(i)} a_{m} q_{m} & \\
\text { s.t. } & g_{i}(l)+\sum_{m \in O(i)} q_{m}=D_{i} & , i \in\{1, \ldots, n\}, \\
& q_{m} \in Q_{m} & , m \in O(i), i \in\{1, \ldots, n\}, \\
& l \in L . &
\end{array}
$$

To simplify the equations, $m$ denotes bands of the piecewise supply functions offered by the generators. $a_{m}$ denotes the offering price of band $m . q_{m}$ is the pre-dispatch quantity to be dispatched from tranche $m . Q_{m}$ implies the capacity constraints of tranche $m$. Note that, firstly each CP node has been formed from the aggregation of several nodes in SPD, and secondly our model has a smaller set of generators than the real SPD, and therefore $O(i)$ is a different set in SPD and CP. Generation from

\footnotetext{
${ }^{4}$ Effectively, demand must always be satisfied.
} 


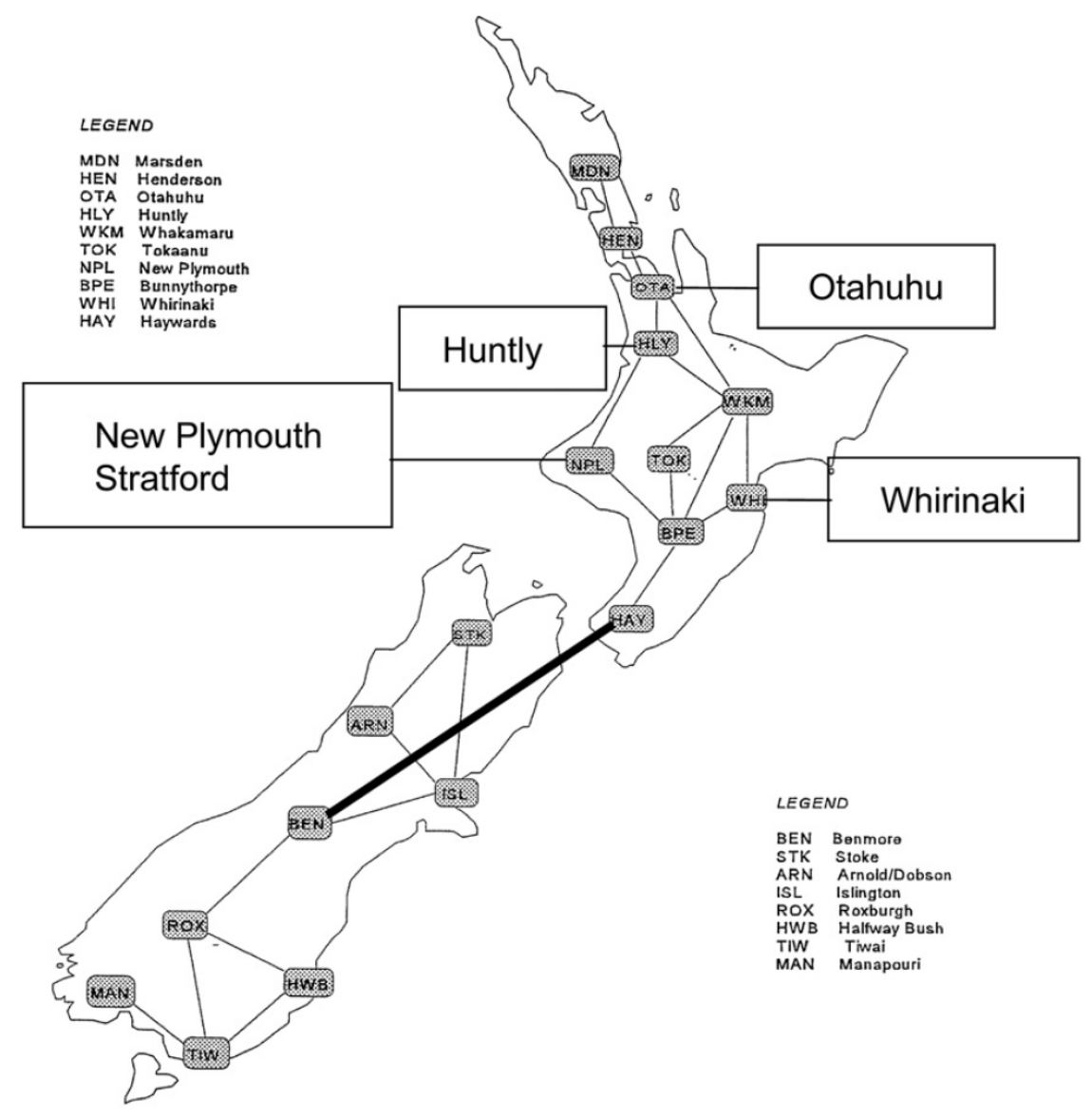

Figure 2: A simplified model of New Zealand electricity network with 18 nodes. HVDC lines between the islands is shown with a bold line [6].

the wind generators are capped by expected production. $L$, as discussed earlier, represents Kirchhoff's Laws and thermal line limits, which is a convex set. $D_{i}$ is demand at node $i$.

\subsection{The stochastic programming model}

Though the SP model is solved before resolution of the uncertainty, it caters for the corrective constraints in the spot market in addition to the constraints of the pre-dispatch problem. The objective 
is to minimize total expected cost of final generation, rather than cost of the pre-dispatch decision.

$$
\begin{aligned}
& \mathrm{SP}: \min _{q, l, y, v} \sum_{i} \sum_{m \in O(i)} \sum_{s} \theta_{s}\left(a_{m} y_{m, s}\right. \\
& \left.+d_{m}^{+}\left(y_{m, s}-q_{m}\right)_{+}+d_{m}^{-}\left(y_{m, s}-q_{m}\right)_{-}\right) \\
& \text {s.t. } g_{i}(l)+\sum_{m \in O(i)} q_{m}=D_{i} \quad, i \in\{1, \ldots, n\} \text {, } \\
& q_{m} \in Q_{m} \\
& , m \in O(i), i \in\{1, \ldots, n\}, \\
& l \in L \\
& g_{i}\left(v_{s}\right)+\sum_{m \in O(i)} y_{m, s}=D_{i} \\
& \begin{array}{r}
, i \in\{1, \ldots, n\}, \\
, s \in\{1, \ldots, S\} \\
, m \in O(i), i \in\{1, \ldots, n\}, \\
, s \in\{1, \ldots, S\} \\
, s \in\{1, \ldots, S\} \\
, m \in O(i), i \in\{1, \ldots, n\}, \\
, s \in\{1, \ldots, S\}
\end{array} \\
& y_{m, s} \in \Upsilon_{m, s} \\
& \begin{array}{r}
, i \in\{1, \ldots, n\}, \\
, s \in\{1, \ldots, S\} \\
, m \in O(i), i \in\{1, \ldots, n\}, \\
, s \in\{1, \ldots, S\} \\
, s \in\{1, \ldots, S\} \\
, m \in O(i), i \in\{1, \ldots, n\}, \\
, s \in\{1, \ldots, S\}
\end{array} \\
& v_{s} \in V_{s} \\
& y_{m, s}-q_{m} \in T_{m}(\tau)
\end{aligned}
$$

In the SP model, $q, l, Q, L, D, a, g$, and $O$ are the same as CP.

- We use $s$ as the index for wind scenarios, and $S$ as the number of wind scenarios.

- $\theta_{s}$ denotes probability of scenario $s$.

- Generation from tranche $m$ in scenario $s$ is denoted by $y_{m, s}$

- $d_{m}^{+}$and $d_{m}^{-}$denote marginal cost of deviation (upward and downward respectively) in the spot market for the generator offering tranche $m$. In our experiments $d_{m}^{+}$, and $d_{m}^{-}$are defined as the inverse of ramp rates $\left(\kappa_{m} \geq 0\right)$ :

$$
\begin{aligned}
d_{m}^{+} & =\frac{\kappa_{m}}{\text { ramp-up rate }}, \\
d_{m}^{-} & =\frac{\kappa_{m}}{\text { ramp-down rate }} .
\end{aligned}
$$

These are decreasing functions of ramp rates. One may raise a question why we have chosen this and not another decreasing function like $\frac{\kappa_{m}}{\text { ramp rate }^{2}}$. The ISO chooses the deviating generators based on the ranking (and not value) of their deviating cost, hence we do not expect to observe a significant difference in the results.

- We use $\left(y_{m, s}-q_{m}\right)_{+}$to denote $\max \left\{y_{m, s}-q_{m}, 0\right\}$ and $\left(y_{m, s}-q_{m}\right)_{-}$to denote $\max \left\{-\left(y_{m, s}-q_{m}\right), 0\right\}$. Both of these are non-negative quantities.

- $\Upsilon_{m, s}$ represent capacity constraint for each tranche and scenario. 
- $v_{s}$ denotes the flow vector in scenario $s$. It is similar to $l$ but is defined for the flow in the spot market.

- The capacity constraint of lines and the Kirchhoff's Laws are implied by $V_{s}$. The spot flow vector of each scenario must satisfy these constraints.

- $T_{m}(\tau)$ is a convex set that includes upward and downward deviation constraints for generator $m$. It is a function of the time between when generator is informed to change output and the time of actual dispatch $(\tau)$.

Note that the only difference between sets $Q_{m}$, and $\Upsilon_{m, s}$ appears for the wind generators. Wind generation in each scenario is capped to the available wind capacity in that scenario by constraint $y_{m, s} \in \Upsilon_{m, s}$.

The last constraint means that SP only chooses qs that are compatible with (and cost-effective for) the spot market. Without this consideration (e.g. in the conventional model), an inappropriately chosen $q$ can result, and deviation in the spot market can be extremely expensive or even impossible (i.e. infeasible).

We use some auxiliary variables that transform this non-linear SP model to an LP problem.

\subsection{The spot market model}

When uncertainty is resolved a corrective action is needed for the conventional mechanism. A spot model minimizes cost of this corrective action using the same offer stack of generators. On the other hand, the SP model considers the spot market and finds corrective actions for the spot market in advance. However, it only considers a limited number of wind scenarios as representatives of all wind possible scenarios. Thus, we still need a spot market for the SP mechanism.

The spot market of both mechanisms uses the same optimization model:

$$
\begin{array}{rlr}
\mathrm{SM}: \min _{q^{r}, l^{r}} & \sum_{i} \sum_{m \in O(i)} a_{m} q_{m}^{r} & \\
\text { s.t. } & g_{i}\left(l^{r}\right)+\sum_{m \in O(i)} q_{m}^{r}=D_{i} & , i \in\{1, \ldots, n\}, \\
& q_{m}^{r} \in Q_{m}^{r} & , m \in O(i), i \in\{1, \ldots, n\}, \\
& l^{r} \in L & \\
& q_{m}^{r}-q_{m} \in T_{m}(\tau) & , m \in O(i), i \in\{1, \ldots, n\},
\end{array}
$$

This problem is similar to CP except in the following aspects.

- The variables and parameters associated with superscript $r$ are post-realization variables and parameters.

- $q^{r}$ and $l^{r}$ are spot market dispatch and flow quantities respectively.

- $Q_{m}^{r}$ is the same as $Q_{m}$, except for the wind generators. For wind generators, $Q_{m}^{r}$ denotes the realized wind generation.

- $q_{m}$ is not a variable but a parameter for this optimization problem, which is a product of the pre-dispatch optimization problem. 
- Other parameters have the same definitions as came before.

SM finds a feasible deviation that minimizes generating cost. The deviations are bound to the predispatch quantities through the last constraint.

\section{Designing experiments to estimate the value of the SPM}

From an optimization perspective, the SPM is preferred to the CM. When there is no strategic bidding, it is quite obvious that the SP mechanism yields higher social welfare. However, implementing the SPM incurs its own expenses. Therefore, we are interested in estimating the benefits of using the SPM for the current wind penetration as well as some possible future penetrations.

\subsection{Current wind penetration}

There are 16 wind generators currently operating in New Zealand. These make up a capacity of 615 MW [9]. Table 1 is taken from New Zealand energy data file ([10]). According to this table, wind generators currently produce about four percent of New Zealand total electricity. The current percentile is not very high. So we expect the current system to work efficiently. To test this claim we compare the current mechanism with the SPM, using the historical data available for the NZEM.

\begin{tabular}{|c|c|c|c|c|c|c|}
\hline Hydro & Gas & Geothermal & Coal & Wind & Bioenergy & Other thermal \\
\hline \hline $56.4 \%$ & $21.2 \%$ & $12.8 \%$ & $4.5 \%$ & $3.7 \%$ & $1.3 \%$ & $0.1 \%$ \\
\hline
\end{tabular}

Table 1: Electricity Generation By Fuel for 2010 [10]

We limit the scope of our analysis to the 6 months between October 2009 and March 2010. We choose this time span, as during this period wind generators have been operating for a while and new wind generation has not been installed.

\subsubsection{Data collecting}

The main input data that we use are available from the CDS. We assume that the NZEM is a competitive market. This means generators do not offer strategically and thus would submit the same offer stack for the SPM. For example, table 2 shows the offer stack of the Huntly station (unit 1) on a Tuesday morning in Feb 2010.

\begin{tabular}{|r|r|r|r|r|r|}
\hline & Band 1 & Band 2 & Band 3 & Band 4 & Band 5 \\
\hline \hline Quantity & 110 & 30 & 30 & 38 & 32 \\
\hline Price & 0 & 85.1 & 145 & 300 & 900 \\
\hline
\end{tabular}

Table 2: The offer stack offered by Huntly (unit 1) on Tuesday, Feb 82010 for time period 8-8:30 am

Generation from co-generation, geothermal plants, and run-of-river hydro plants is usually hard to control. We fix generation from these sources to their historical dispatch figures, which are available in the CDS. The other generators are the offering generators. Table 3 summarizes the offering units in our model and their capacity. 


\begin{tabular}{|l|l|l|}
\hline & Number of units & Total capacity (MW) \\
\hline \hline Hydro & 21 & 4,518 \\
\hline Thermal & 12 & 2,827 \\
\hline Wind & 3 & 236 \\
\hline
\end{tabular}

Table 3: Offering Units in our model and their capacity

\begin{tabular}{ccc}
\hline Number of Units & Ramping Rate $(\mathrm{MW} / \mathrm{h})$ & Capacity $(\mathrm{MW})$ \\
\hline \hline 3 & 240 & 360 \\
5 & 300 & 1,204 \\
1 & 390 & 396 \\
1 & 480 & 50 \\
1 & 600 & 387 \\
1 & 990 & 430 \\
\hline
\end{tabular}

Table 4: Ramping rates for the thermal units

We use the ramp rates of generators to construct our ramping constraints (in the SP and SM models). They are also used to determine the marginal cost of deviation in the objective function of the SP model. Assume that the ISO requests extra generation from the spot market about 10 minutes before real dispatch. Then, the maximum increase that a generator is able to handle is equal to $\frac{\text { Ramp up rate } * 10}{60}$. These constraints are mainly imposed upon the thermal generators. Ramp rates for the thermal generators are listed in table 4 .

Demand data is aggregated into 18 nodes as depicted in fig. 2. We inflate demand by a factor (e.g. $5 \%)$ to account for the loss of electricity in the omitted lines in the simplification process.

\subsubsection{Scenario generation}

For the SP model, we need to produce several wind scenarios. These scenarios shape a discrete distribution for the wind production in the spot market. Each scenario determines a wind generation quantity and the probability associated with it. The SP model is assumed to be solved 2 hours before actual dispatch. This means we need to generate wind scenarios for every time period two hours ahead of actual dispatch. To produce a distribution for the wind production two hours later, different factors such as current wind production or current wind direction can be used. We build the distribution based on the wind generation now. We use some quantile-type models for producing the wind scenarios. These models predict quantiles of the conditional distribution of the random variable (i.e. wind production two hours later) using currently available information. For a detailed description of this method, see [11].

There are two main sites in New Zealand with high amounts of electricity production from wind generators, one in the North Island and one in the South Island. As wind farms in the North Island are located closely, we consider a single random variable for the total generation from these wind farms. This reduces the complexity of the model greatly, because we do not need to consider the correlation between wind production in a joint distribution function. On the other hand, over the 


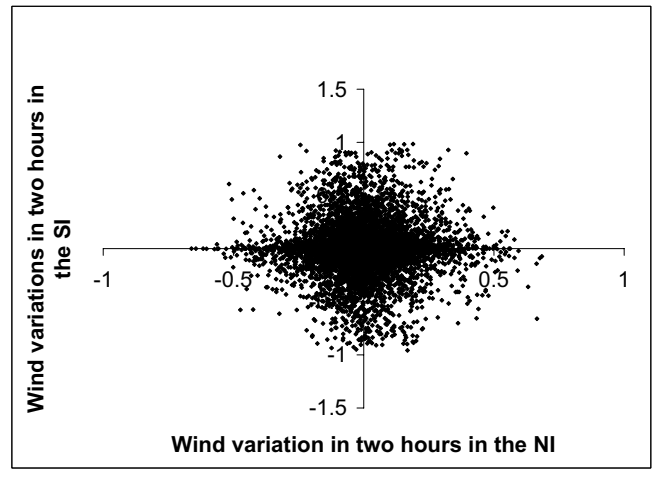

Figure 3: The South Island (SI) and North Island (NI) wind variations (over two hours) for each time period (from 31/3/2008 to 31/3/2010); Each point represents one time period.

time span within which we are interested, White Hill is the only major wind farm in the South Island, which is located far from the North Island plants. Figure 3 shows that there is little correlation between changes in wind power in the South Island and in the North Island. The correlation between these variations for 2 years from March 2008 to March 2010 is -0.002 . Thus, we can assume that wind variations in the North Island is independent from those of the South Island, and the joint distribution can be represented as

$$
f_{N I, S I}\left(w^{N I}, w^{S I}\right)=f_{N I}\left(w^{N I}\right) . f_{S I}\left(w^{S I}\right) .
$$

We extract 6 scenarios for each of the islands for each time period. This makes a total of 36 wind scenarios. In this section, we only present our methodology for producing wind scenarios for the North Island, as South Island scenarios can be obtained using the same methodology.

Wind production two hours later, which is the random variable here, is defined as a percentage of the total capacity, thus it ranges between 0 and 1 . This is defined for every time period, with the information available two hours before the time period.

The electricity generation from power stations Te Apiti, and Tararua I/II between January 2005 and March 2010 is used as the raw data for the quantile-type model in the North Island. A crossvalidation exercise, in which some of the data are compared to the predictions from the quantile models of the rest, can assure that our quantile models perform as intended [11]. The time periods in which one or more of these power stations were not available with full capacity is excluded from these data. This makes a total of about 5 years of input data.

Each point in fig. 4 represents the wind production in a time period as a function of wind production two hours before the time period. We use the method of [11] for constructing our quantile models. The $\gamma$-quantile $Q_{\gamma}(0<\gamma<1)$ of the distribution is a linear combination of the basis functions $b_{i}(w)$.

$$
Q_{\gamma}(w) \approx \sum_{i=0}^{n_{w}} \beta_{i}(\gamma) b_{i}(w)
$$

Here, $n_{w}$ is chosen 5 , and $b_{0}(w)$ is assumed to be 1 . The aim is to find the appropriate $\beta_{i}(\gamma)$ coefficients. For this purpose, we use the quantreg package of statistical language R. The red curves in fig. 4 represent 5 equally-likely estimated quantile scenarios. 


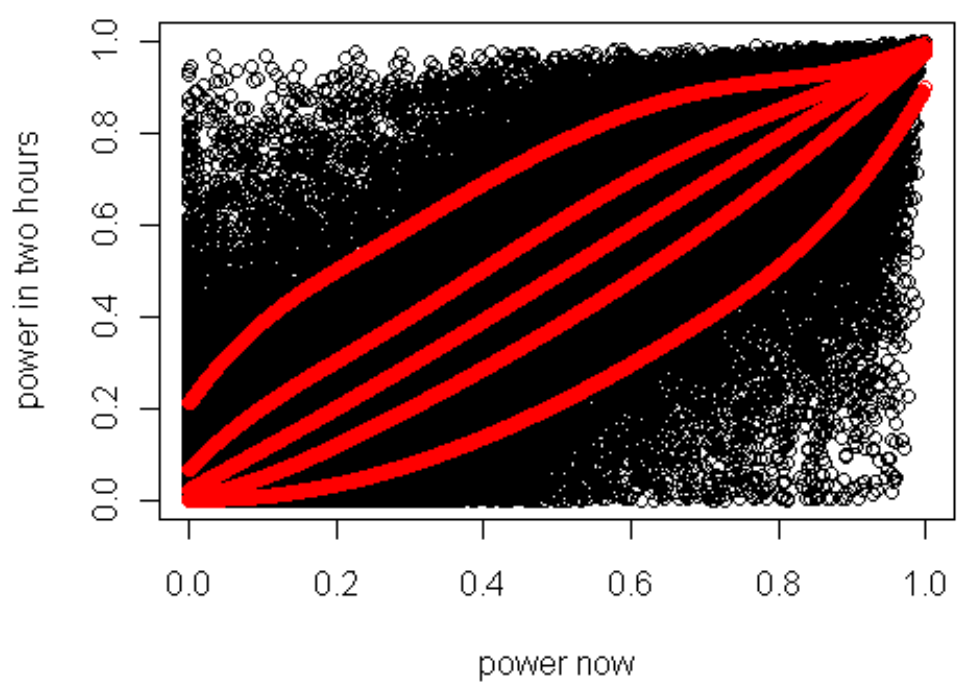

Figure 4: NI non-zero Scenarios

The time periods with zero production are excluded from this chart (i.e. fig. 4), because zero production may be a result of no wind at all or any wind speed bellow the turbine cut-in speed, or above the turbine cut-out speed. Thus, we define a separate zero production scenario with the probability

$$
\theta_{1}^{N I}(w)=\left\{\begin{array}{ll}
\frac{g_{0}^{N I}}{h_{0}^{N I}} & \text { if } w=0 \\
\rho^{N I} e^{\eta^{N I} w} & \text { if } w>0
\end{array},\right.
$$

where,

- The index 1 represents the scenario with zero wind production in two hours, if the observed wind production is $w$.

- The observed wind production is denoted by $w$, i.e. the wind production two hours before the time period for which we construct the distribution function.

- $h_{0}^{N I}$ denotes the total number of time periods with zero observed wind production from wind generators in the North Island.

- $g_{0}^{N I}$ denotes the number of time periods with zero observed production (i.e. zero production two hour before dispatch) and zero actual production.

- $\rho^{N I}$ and $\eta^{N I}$ are the coefficients of the fitted exponential function to the 2-tuples $\left\{\frac{g_{i}^{N I}}{h_{i}^{N I}}, \frac{i}{100}\right\}$ for $i$ in $\{1,2, \ldots, 100\} . h_{i}^{N I}$ is the number of time periods with an observed wind production in $\left[\frac{i-1}{100}, \frac{i}{100}\right)$, and $g_{i}^{N I}$ is the number of these time periods with zero production in two hours. In 
other words, $\frac{g_{i}^{N I}}{h_{i}^{N I}}$ is the probability of zero wind production in two hours if the current wind production is in $\left[\frac{i-1}{100}, \frac{i}{100}\right)$. These 2-tuples are shown in fig. 5 . We assign this probability to the $\mathrm{x}$-axis value of $\frac{i}{100}$. We fit an exponential function through these points as an approximation of the probability of zero production in two hours for a given current production. For the specific case of New Zealand electricity market the estimated probabilities of the first scenario are

$$
\begin{aligned}
\theta_{1}^{N I} & =0.25 e^{-15.8 w}, \\
\theta_{1}^{S I} & =0.24 e^{-10.6 w} .
\end{aligned}
$$

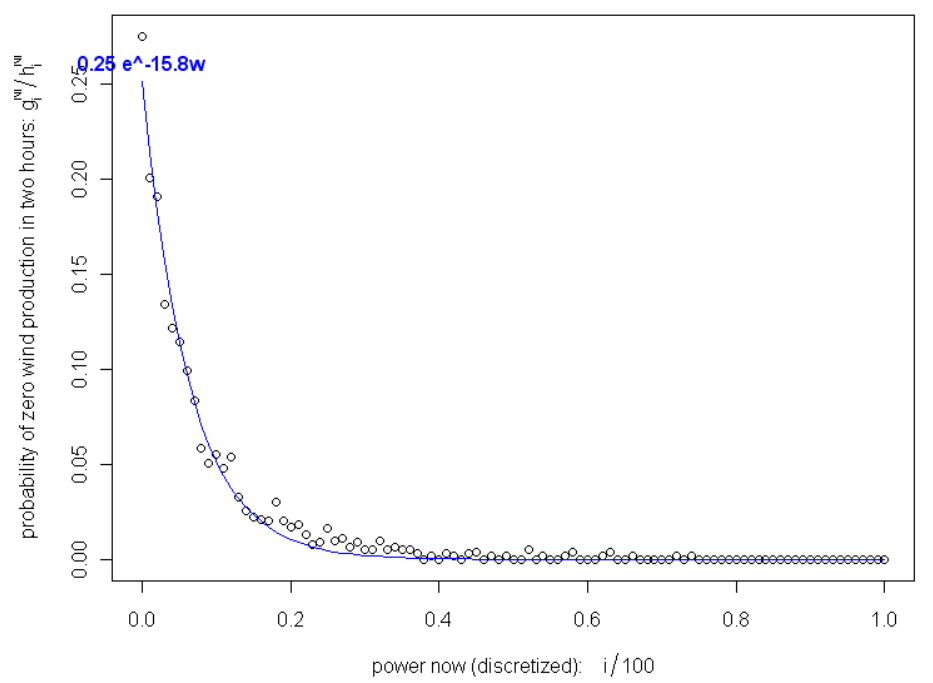

Figure 5: Discretized probability of no wind production as a function of the observed wind production

As discussed earlier the other 5 scenarios are equally likely. To find these scenarios, we can divide the historical data in fig. 4 into 5 equally-likely bands. The boundaries of these bands are $\frac{j}{5}$-quantiles ( $j$ in $\{0,1, \ldots, 5\}$ ) of the data. For each band a middle point can represent the predicted quantity for the scenario. This middle points are the medians of the bands which can also be represented as $\frac{\frac{j}{5}+\frac{j+1}{5}}{2}$-quantiles $(j$ in $\{0,1, \ldots, 4\})$. Therefore, if we represent the predicted production for scenario $s$ with $\gamma(s)$-quantile of the distribution, $\gamma(s)$ is as follows.

$$
\gamma(s)=\frac{2(s-1)-1}{10} \quad, 2 \leq s \leq 6
$$

Table 5 lists the 6 predicted wind scenarios and the probability of each scenario for the North Island wind generation, if the current wind generation is $w$.

With a similar method we generate 6 scenarios for the South Island. The scenario set is the Cartesian product of North Island and South Island scenarios with the probability

$$
\theta_{s}=\theta_{s^{N I}} \theta_{s^{S I}} \quad, s=\left\{s^{N I}, s^{S I}\right\} .
$$

The $\mathrm{R}$ code used for scenario generation is available on request. 


\begin{tabular}{|c|c|c|}
\hline Scenario & Predicted production & Probability \\
\hline$s=1$ & 0 & $\theta_{1}^{N I}(w)$ \\
$s, 2 \leq s \leq 6$ & $Q_{\gamma(s)}(w)$ & $\frac{1-\theta_{1}^{N I}(w)}{5}$ \\
\hline
\end{tabular}

Table 5: North Island scenarios

\subsection{More wind penetration}

New Zealand is a country with good potential for wind generation [12]. The growth rate of wind energy has been around $27 \%$ in the recent past years [10]. At the moment, several projects are under consideration to increase wind generation in NZEM (e.g. see [13]). Thus, higher wind penetration is very likely in the near future.

We are interested in designing different experiments to see the effect of higher wind integration in New Zealand. Due to lack of information, it is difficult to construct a scenario set for future wind integration. Thus, the only possible experiment is to inflate wind generation by a factor for the North Island and South Island generators. Inflating wind capacity in one place opposed to what happens in reality (i.e. wind integration dispersed around the country) has a higher variability in wind generation. Thus, it is more difficult to deal with the uncertainty in such a situation. Therefore, our analysis provides pessimistic results for the CM.

For each experiment, we use the scenario set produced for the current wind integration. Then, we inflate each wind production scenario in the North Island (South Island) with the coefficient $K^{N I}$ $\left(K^{S I}\right)$. Therefore, the scenario set for the NI can be represented as of table 6. South Island scenarios are constructed similarly.

\begin{tabular}{|c|c|c|}
\hline Scenario & Predicted production & Probability \\
\hline$s=1$ & 0 & $\theta_{1}^{N I}(w)$ \\
$s, 2 \leq s \leq 6$ & $K^{N I} Q_{\gamma(s)}(w)$ & $\frac{1-\theta_{1}^{N I}(w)}{5}$ \\
\hline
\end{tabular}

Table 6: North Island scenarios for higher wind integration

The probability of each scenario is assumed the same as the current wind integration, i.e.

$$
\theta_{s}=\theta_{s^{N I}} \theta_{s^{S I}} \quad, s=\left\{s^{N I}, s^{S I}\right\} .
$$

We also inflate actual wind production with the same factor.

\section{$5 \quad$ Experiments and Results}

In this section, we compare the solutions to our mechanism (SPM and CM) for all half-hourly trading periods over an extended period of six months (from 1/10/2009 to 31/03/2010). Subsections 5.1 and 5.2 compare the two models for the current and future wind integration, using a fixed value of $\kappa_{m}=0.01$ for all generating units. Subsection 5.3 discusses the sensitivity of our results to $\kappa_{m}$. In all experiments, the Conventional Model assumes a single wind scenario corresponding to the persistence wind forecast, i.e. no change in wind generation over the time horizon. 


\begin{tabular}{|l|r|r|}
\hline & Cost $(\$)$ & Saving $(\$)$ \\
Date & CM & SPM: $\kappa=0.01$ \\
\hline 07.10 .09 & 754,957 & -7.20 \\
08.10 .09 & 675,584 & 60.53 \\
09.10 .09 & $1,244,704$ & 0.00 \\
10.10 .09 & 716,098 & 0.00 \\
11.10 .09 & 255,537 & 0.00 \\
12.10 .09 & 241,187 & 300.84 \\
13.10 .09 & 305,166 & 217.35 \\
27.10 .09 & 889,209 & 81.47 \\
\hline
\end{tabular}

Table 7: Cost of the conventional mechanism and saving of SP mechanism for different deviating cost parameters for selected days

\begin{tabular}{|r|r|}
\hline Days with improvement (\%) & 16 \\
Days with decline (\%) & 4 \\
\hline Average of Saving (\$/day) & 5.91 \\
Max of Saving (\$/day) & 300.84 \\
Min of Saving (\$/day) & -548.15 \\
\hline
\end{tabular}

Table 8: A summary of the saving of the SP mechanism for current wind integration.

\subsection{Experiment 1: Current wind integration}

Table 7 shows the cost saving of the SPM for eight typical days. This is the difference in realized costs of generation according to our models with wind generation as it occurred historically ${ }^{5}$. As ramping costs have not been included in the original SPD and our model of it (CM), our cost criterion does not include deviation costs. This way we ensure that we do not advantage the SPM. The cost saving of the SPM for 12/10/2009 is $\$ 300$. On the other hand, the SPM increases the total cost of generation in some days. For example, the cost saving of SPM is $\$-548$ for $2 / 10 / 2009$. Note that we do not always expect the SPM to improve the cost of generation. The solution to the SPM minimizes only the expected cost for different scenarios.

The cost difference of $\$ 300$ in 12/10/2009 can be attributed mainly to the difference in dispatch in period 20, where Huntly pre-dispatch quantity is lower in the SP mechanism. Huntly is an expensive thermal generator and is not very flexible. The spot market needs the Huntly generator to reduce power as much as it can. It deviates up to its ramping constraint in both SP and Conventional mechanisms. Therefore, the final generation from Huntly (which is offered at price 48) is lower in the SP mechanism, and instead the hydro generator Maraetai (which is offered at price 20) is used.

Table 8 shows a summary of the cost saving of the SP mechanism for the current wind integration. An average saving of $\$ 5.91$ per day is achieved in this case.

\footnotetext{
${ }^{5}$ Another option is to compare these two mechanism based on the expected cost of generation (see [14]). Our cost comparison, however, is based directly on actual wind generation rather than the constructed scenarios we have used for the model. Having run the numerical experiments for six months does make the overall result much like an expected value; it could be argued that this is actually better than computing expected values with respect to the small number of scenarios used in the stochastic program.
} 


\subsection{Experiment 2: Future wind integration}

As discussed in subsection 4.2, we assume that future wind power developments occurs at the same locations as existing wind farms. This approach has more variability in comparison with a case with wind dispersed all around the country. Thus, the performance of our mechanisms in our analysis (specially CM) is estimated to be worse in comparison with a real situation with the same volume of wind integration.

\begin{tabular}{|r|r|r|r|r|}
\hline & Cost (\$) & Cost (\$) & Saving (\$) & Saving (\%) \\
Date & CM & SPM & SPM & SPM \\
\hline 7.10 .09 & 87,369 & 92,232 & $-4,863$ & -5.57 \\
8.10 .09 & 15,033 & 12,571 & 2,462 & 16.38 \\
9.10 .09 & 46,329 & 41,823 & 4,506 & 9.73 \\
10.10 .09 & 6,488 & 6,488 & 0 & 0.00 \\
11.10 .09 & 82,834 & 82,834 & 0 & 0.00 \\
12.10 .09 & 3,618 & 3,618 & 0 & 0.00 \\
13.10 .09 & 0 & 0 & 0 & $\mathrm{NA}$ \\
27.10 .09 & 7,139 & 5,239 & 1,900 & 26.61 \\
\hline
\end{tabular}

Table 9: Cost saving of SPM with $K^{\mathrm{NI}}=K^{\mathrm{SI}}=5$ for selected days

We use parameters $K^{\mathrm{NI}}$ and $K^{\mathrm{SI}}$ to inflate wind generation in all scenarios. As the current wind integration is around $4 \%, K^{\mathrm{NI}}=K^{\mathrm{SI}}=5$ can represent a situation with $20 \%$ wind integration. Table 9 shows this situation for selected days.

The results in table 9 are very different from those of table 7 . The savings in dollar are much higher in this case. The other interesting observation is the difference between the cost of generation in the cases of $4 \%$ and $20 \%$ integration.

A comparison between table 10 and table 8 shows that the average saving from the SPM has increased from $\$ 6$ to $\$ 3,518$. In situations with more wind integration, the SPM mechanism has a better performance. In other words, the CM gradually becomes an inefficient market clearing mechanism when wind integration increases in the system.

\subsection{Experiment 3: The choice of deviation cost coefficient $\left(\kappa_{m}\right)$}

The SP problem, defined in subsection 3.2, has two mechanisms to control the deviations from the pre-dispatch quantities, and to ensure a feasible and optimal (i.e. in expectation) solution. Firstly, constraint $y_{m, s}-q_{m} \in T_{m}(\tau)$, ensures the feasibility of the spot deviations for all scenarios. Secondly, the cost of deviation in the objective function causes the solution to SP to favour the generators with lower cost of deviation to deviate in the spot market. The deviation penalty also helps in finding a centrally located solution in the case that several optimal solutions exist. This reduces the variability of improvement or decline of the SPM. Thus, this penalty can reduce the risk. On the other hand, the deviation penalties have a negative effect on the primary objective (i.e. minimizing total cost of generation), especially if they are not chosen to be small enough. The deviation penalties $d_{m}^{+}$ and $d_{m}^{-}$are defined in (2) and (3) as functions of $\kappa_{m}$, which can take any non-negative value. In this experiment, we try to estimate the negative and positive effects of the deviation penalty in the objective function. 


\begin{tabular}{|r|r|}
\hline Days with improvement (\%) & 65 \\
Days with decline (\%) & 9 \\
\hline Average of Saving (\$/day) & 3,518 \\
Max of Saving (\$/day) & 55,049 \\
Min of Saving (\$/day) & -4863 \\
\hline
\end{tabular}

Table 10: Saving of the SP mechanism for $K^{\mathrm{NI}}=K^{\mathrm{SI}}=5$ (from $1 / 10 / 2009$ to $31 / 03 / 2010$ )

\begin{tabular}{|c|r|r|r|r|r|}
\hline Date & Cost $(\$)$ & Saving (\$) & Saving (\$) & Saving (\$) & Saving (\$) \\
CM & SP: $\kappa=0$ & SP: $\kappa=0.01$ & SP: $\kappa=1$ & SP: $\kappa=100$ \\
\hline 07.10 .09 & 754,957 & 41.40 & -7.20 & -7.20 & -7.20 \\
08.10 .09 & 675,584 & 90.85 & 60.53 & 60.53 & 60.53 \\
09.10 .09 & $1,244,704$ & 30.00 & 0.00 & 0.00 & 0.00 \\
10.10 .09 & 716,098 & 0.00 & 0.00 & 0.00 & 0.00 \\
11.10 .09 & 255,537 & 817.01 & 0.00 & 0.00 & 0.00 \\
12.10 .09 & 241,187 & $3,661.04$ & 300.84 & 203.49 & 219.19 \\
13.10 .09 & 305,166 & -960.24 & 217.35 & 217.35 & 217.35 \\
27.10 .09 & 889,209 & 184.99 & 81.47 & 81.47 & 63.63 \\
\hline
\end{tabular}

Table 11: Cost of the conventional mechanism and saving of SP mechanism for different deviating cost parameters for selected days

Table 11 shows amount of saving from the SP mechanism for different $\kappa$ values for some selected days. The first observation is that if $\kappa>0$, the saving is quite similar, and slightly better for smaller $\kappa$ values (e.g. see 12.10 .09 and 27.10.09). This can be a result of the negative effect of larger deviating penalties on the primary objective function. However, the cases with $\kappa=0$ and $\kappa>0$ seem very different. Thus, we can compare the case with $\kappa=0$, and $\kappa=0.01$, for more days. Table 12 shows a summary of the results of 100 days for these two cases. As expected the variability in the case with $\kappa=0$ is much higher than the case with $\kappa=0.01$. This variability (e.g. a day with $\$-4,284.87$ ) also has caused the average saving to be less than the case with $\kappa=0.01$.

\begin{tabular}{|r|r|r|}
\hline & SP: $\kappa=0$ & SP: $\kappa=0.01$ \\
\hline Days with improvement (\%) & 22 & 16 \\
Days with decline (\%) & 4 & 4 \\
\hline Average of Saving (\$/day) & 3.67 & 5.91 \\
Max of Saving (\$/day) & $3,661.04$ & 300.84 \\
Min of Saving (\$/day) & $-4,284.87$ & -548.15 \\
\hline
\end{tabular}

Table 12: Saving of the SP mechanism for 100 days (from 1/10/2009 to 8/01/2010)

In sum, in the previous sections we used $\kappa=0.01$, as firstly it is larger than zero and provides less risky solutions, and secondly, it is small enough and has little negative effect on the primary objective function. 


\section{Conclusion}

In this paper, we first presented a simplified version of the NZEM. We also presented a stochastic market clearing mechanism for New Zealand electricity market.

The stochastic market clearing mechanism calls for extra input data, such as wind scenarios and cost of deviations. These parameters do not exist in the current New Zealand market. Therefore, we have used some statistical methods to estimate these parameters.

Also, we designed experiments to quantify the value of stochastic optimization for the current market environment, as well as possible future environments with larger wind integration.

Our analysis shows that the saving of a stochastic market clearing is not significant for the current New Zealand electricity market. However, when wind integration increases to 20\%, an estimated daily average saving of $\$ 3518$ is achieved for a six month period under investigation. In other words, for large-scale wind integration the conventional dispatch might not be efficient enough, and it is essential to consider migrating to a more efficient mechanism like stochastic market clearing mechanism.

\section{References}

[1] S. Wong and J. Fuller, "Pricing energy and reserves using stochastic optimization in an alternative electricity market," Power Systems, IEEE Transactions on, vol. 22, no. 2, pp. 631-638, 2007.

[2] G. Pritchard, G. Zakeri, and A. Philpott, "A Single-Settlement, Energy-Only electric power market for unpredictable and intermittent participants," Operations Research, Apr. 2010.

[3] F. Bouffard, F. Galiana, and A. Conejo, "Market-clearing with stochastic security-part i: formulation," Power Systems, IEEE Transactions on, vol. 20, no. 4, pp. 1818-1826, 2005.

[4] "New zealand celebrates global wind day for the first time - new zealand wind energy association." http://windenergy.org.nz/component/content/article/117-2011-media-releases/247-newzealand-celebrates-global-wind-day-for-the-first-time.

[5] "Global wind energy outlook," tech. rep., GWEC, 2010.

[6] A. Philpott, Z. Guan, J. Khazaei, and G. Zakeri, "Production inefficiency of electricity markets with hydro generation," Utilities Policy, vol. 18, pp. 174-185, Dec. 2010.

[7] NZIER, "The markets for electricity in new zealand," tech. rep., 2007.

[8] T. Alvey, D. Goodwin, X. Ma, D. Streiffert, and D. Sun, "A security-constrained bid-clearing system for the new zealand wholesale electricity market," IEEE Transactions on Power Systems, vol. 13, pp. 340-346, May 1998.

[9] "NZ wind farms - new zealand wind energy association." http://windenergy.org.nz/nz-windfarms/nz-wind-farms.

[10] "New zealand energy data file," tech. rep., Ministry of Economic Development, 2011.

[11] G. Pritchard, "Short-term variations in wind power: Some quantile-type models for probabilistic forecasting," Wind Energy, vol. 14, no. 2, pp. 255-269, 2011. 
[12] P. J. Edwards, "Wind energy resource studies in new zealand," Solar $\&$ wind technology, vol. 7, no. 1, p. 9-14, 1990.

[13] "Brooklyn wind turbine - new zealand wind energy association." http://www.windenergy.org.nz/nz-wind-farms/operating-wind-farms/brooklyn.

[14] F. Maggioni and S. W. Wallace, "Analyzing the quality of the expected value solution in stochastic programming," Annals of Operations Research, vol. 200, no. 1, pp. 37-54, 2012. 